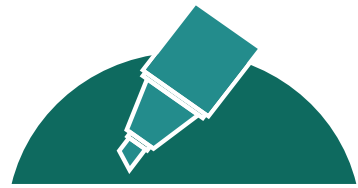 \\ Guías \\ prácticas
}

Apropiación social del conocimiento

Generación de contenidos impresos https://repository.ucc.edu.co/handle/20.500.12494/7375 N. ${ }^{0} 10$, noviembre de 2021 doi: https://doi.org/10.16925/gcgp.33

\section{TOMA DE RELACIÓN BICONDILO MAXILAR}

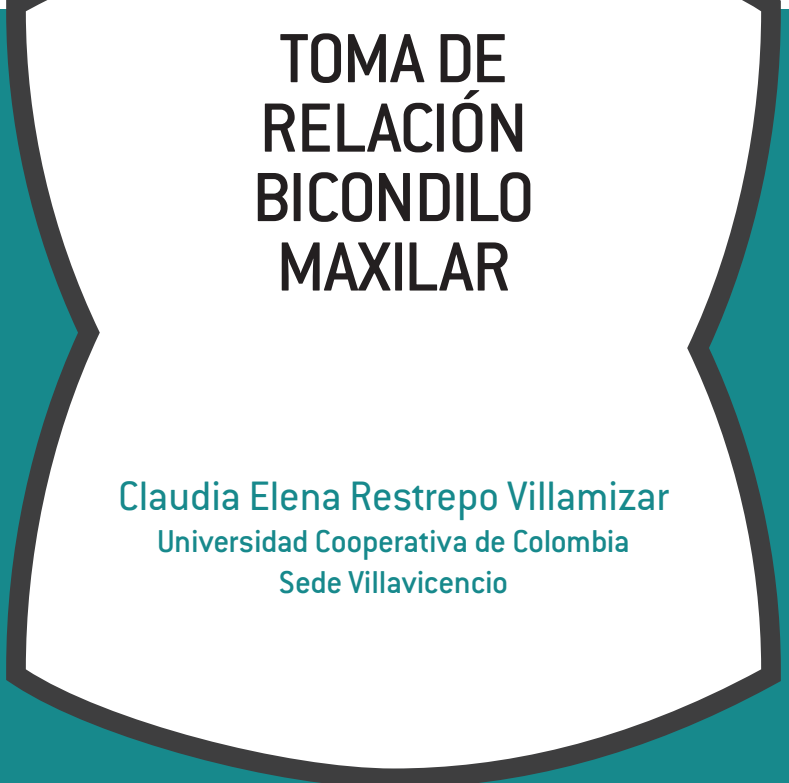




\section{ACERCA DE LA AUTORA}

Claudia Elena Restrepo Villamizar, odontóloga especialista en Salud Familiar, en proceso de maestría en educación, profesora instructora del programa de Odontología, Universidad Cooperativa de Colombia, sede Villavicencio, Colombia.

Correo electrónico: claudiae.restrepo@campusucc.edu.co

Orcid https://orcid.org/0000-0002-8308-5913

\section{CÓMO CITAR ESTE DOCUMENTO}

Restrepo Villamizar CE. Toma de relación bicondilo maxilar (Generación de contenidos impresos N. $\left.{ }^{\circ} 10\right)$. Ediciones Universidad Cooperativa de Colombia, 2021. doi: https://doi. org/10.16925/gcgp.33

NOTA LEGAL

El presente documento de trabajo ha sido incluido dentro de nuestro repositorio institucional como Apropiación social de conocimiento por solicitud del autor, con fines informativos, educativos o académicos. Asimismo, los argumentos, datos y análisis incluidos en el texto son responsabilidad absoluta del autor y no representan la opinión del Fondo Editorial o de la Universidad.

\section{DISCLAIMER}

This coursework paper has been uploaded to our institutional repository as Social Appropriation of Knowledge due to the request of the author. This document should be used for informational, educational or academic purposes only. Arguments, data and analysis included in this document represent authors' opinion not the Press or the University.

(c) (1) () () Este documento puede ser consultado, descargado o reproducido desde nuestro repositorio institucional (http://repository. ucc.edu.co/handle/20.500.12494/7369) para uso de sus contenidos, bajo la licencia de Creative Commons Reconocimiento-NoComercial-SinObraDerivada 4.0 Internacional. http://creativecommons.org/licenses/by-nc-nd/4.0/ 


\section{TABLA DE CONTENIDO}

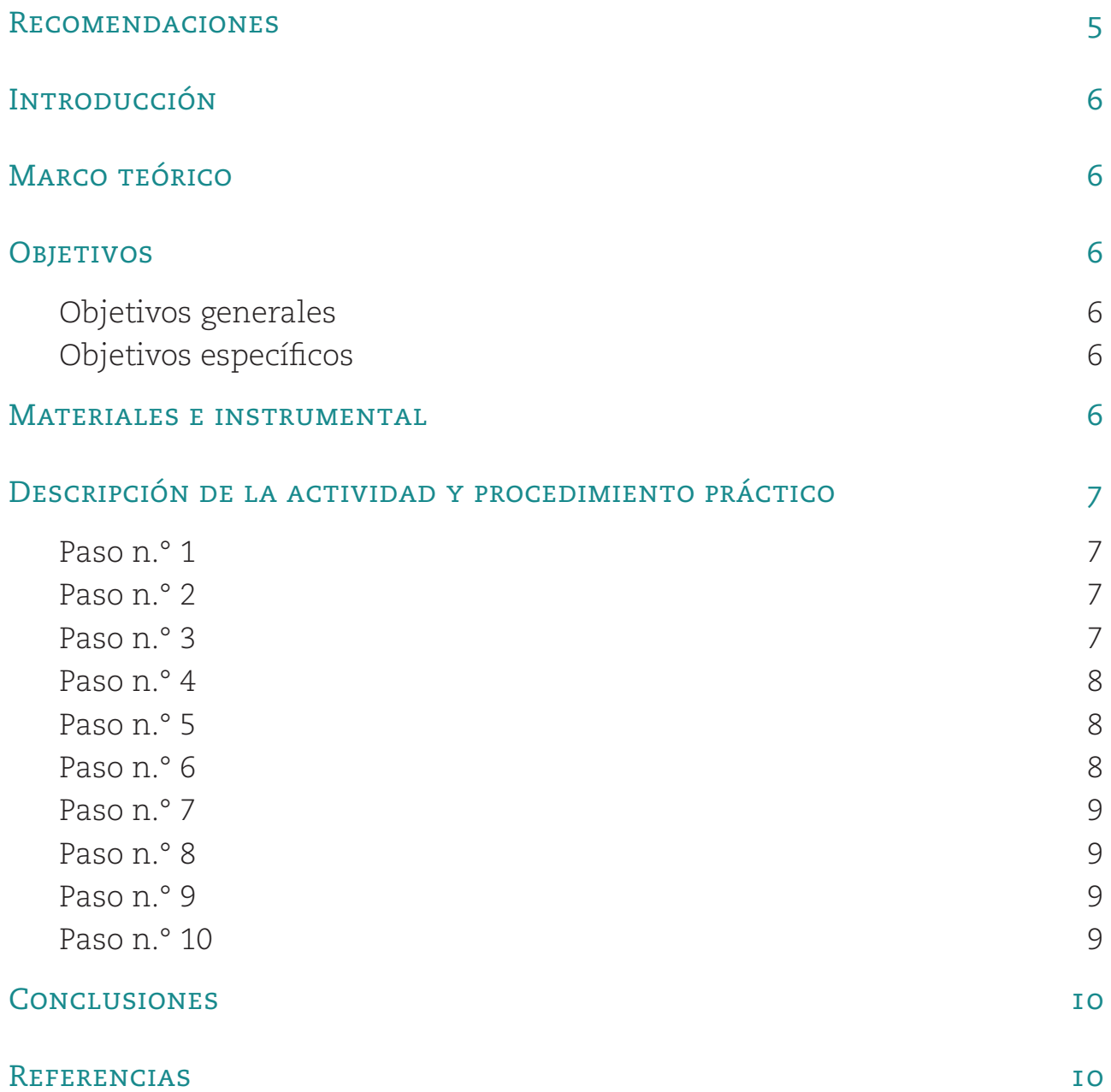




\title{
10 TOMA DE RELACIÓN BICONDILO
MAXILAR
}

\author{
Claudia Elena Restrepo Villamizar
}

\section{Resumen}

El objetivo de la guía es ayudar al estudiante a tener conocimiento del paso a paso en la toma de la relación bicondilo maxilar para que, posteriormente, pueda representar las posiciones mandibulares necesarias para un diagnóstico, pronóstico y tratamiento del paciente; lo anterior teniendo en cuenta cada uno de los detalles al momento de realizar la toma en el consultorio odontológico o en el área de práctica clínica.

Palabras clave: consultorio odontológico, oclusión dental, registro de la relación maxilomandibular, seguridad del paciente. 


\section{Recomendaciones}

- Es importante que para la toma de la relación bicondilo maxilar, el estudiante tenga conocimiento sobre la manera de manipular cada uno de los elementos que componen el articulador, reconozca la función y el uso de cada una de las partes para así garantizar el éxito final del montaje.

- Conocer las condiciones para el uso de cada uno de ellos.
- Seguir las indicaciones del profesor.

- Preparación del paciente explicándole lo que se le va a realizar.

- Utilizar productos certificados.

- Previa toma de impresiones en alginato.

- Posterior obtención de modelos. 


\section{Introducción}

El articulador es el aparato de mayor importancia para poder llevar a cabo la toma de un registro, montaje y movimientos mandibulares; este es un aparato mecánico que representa la articulación temporomandibular y los componentes de los maxilares, al que pueden incorporarse modelos tanto del maxilar superior como de la mandíbula y simular movimientos [1].

La toma de la relación bicondilo maxilar busca la verdadera posición de la mandíbula en relación con la cavidad glenoidea y los demás componentes que hacen parte de la articulación temporomandibular (ATM); lo anterior tiene como fin el diagnóstico y el tratamiento adecuados, así como una rehabilitación exitosa.

Mostraremos el paso a paso para la toma del registro bicondilo maxilar de manera que el estudiante logre de una manera clara y precisa la toma del registro. La guía va con toma de fotos que va explicando la teoría y para que así logren alcanzar la competencia requerida como es: "Analiza las relaciones oclusales en normalidad de la dentición permanente”.

\section{Marco teórico}

Para lograr una toma de relación bicondilo maxilar se necesita de un adecuado articulador que contenga el arco facial, tenedor u horquilla y Nation. En relación con los datos históricos, Phillip Pfaff, en 1756, describió lo que fue el primer articulador de yeso; por su parte, Jean Baptiste Gariot 1805 (Francia) inventó el primer articulador de bisagra simple [2].

El doctor Gysi estudio la oclusión y demostró que los cóndilos dirigen el camino que siguen los dientes en movimientos extrusivos de la mandíbula. Fue el primero que utilizó un instrumento para determinar la dentición intermaxilar céntrica y trazó las proyecciones externas de los desplazamientos de los cóndilos [3].

En 1856, comenzó el uso de la godiva o compuesto de modelar, material usado hoy en día para tomar registros de mordida durante la toma de la relación bicondilo maxilar. Como vemos, con el tiempo, se han venido modernizando los diferentes conceptos relacionados con la oclusión y se han diversificado las técnicas utilizadas para lograr un tratamiento exitoso, además, de la destreza y conocimiento requeridos para realizar este ejercicio.

\section{Objetivos}

\section{OBJETIVOS GENERALES}

- Demostrar la técnica de toma de relación bicondilo maxilar para brindar mayor conocimiento a los estudiantes.

- Explicarles a los estudiantes de odontología todo lo concerniente a una buena toma de relación bicondilo maxilar para que cuenten con herramientas precisas para el adecuado manejo del articulador y garantizar relaciones oclusales óptimas; lo anterior está basado en la literatura científica.

\section{OBJETIVOS ESPECÍFICOS}

- Enumerar el paso a paso hasta lograr perfeccionar la técnica de toma de relación bicondilo maxilar.

- Mostrar la agilidad del estudiante al realizar la práctica sobre su paciente.

\section{Materiales e instrumental}

- Arco facial

- Tenedor u horquilla 
- Nasion

- Sistema de ajuste de la horquilla

- Ramas del arco facial

- Godiva

- Mechero

- Babero

- Tapabocas

- Gorro

- Bata

- Elementos de protección general como parte de la seguridad del paciente y del operador

\section{Descripción de la actividad y procedimiento práctico}

\section{PASO N. ${ }^{0} 1$}

Tenga listos los materiales, como se presenta en la figura 1.

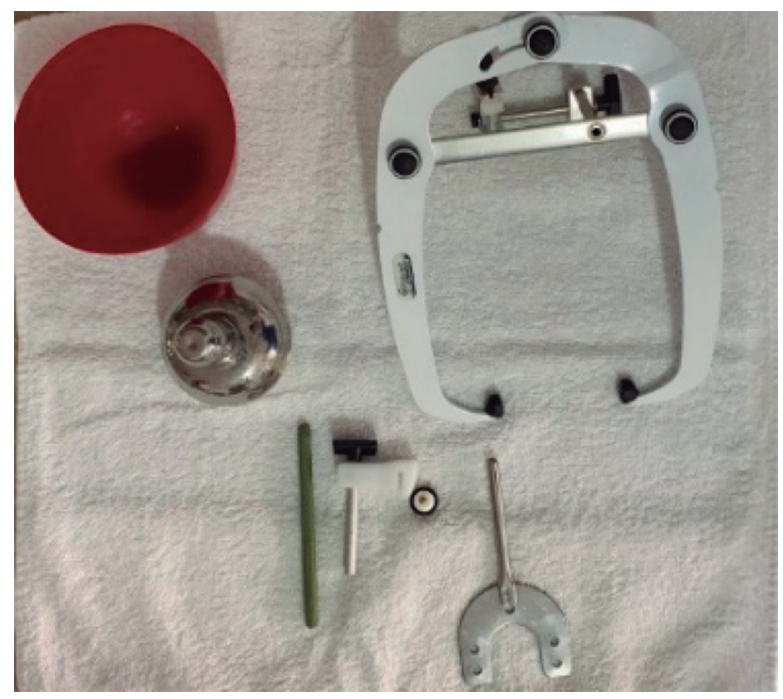

FIGURA 1. Materiales.

Fuente: elaboración propia.

\section{PASO N. ${ }^{\circ} 2$}

Tomamos la horquilla y le agregamos godiva de baja fusión previamente calentada en el mechero, con el fin de que su aspecto sea fluido. Se coloca en tres puntos específicos, a nivel de anteriores y en posteriores, como se ve en la figura 2. Se puede usar cualquier otro material termoplástico que ofrezca estabilidad y que garantice que queden registradas las caras oclusales y bordes incisales.

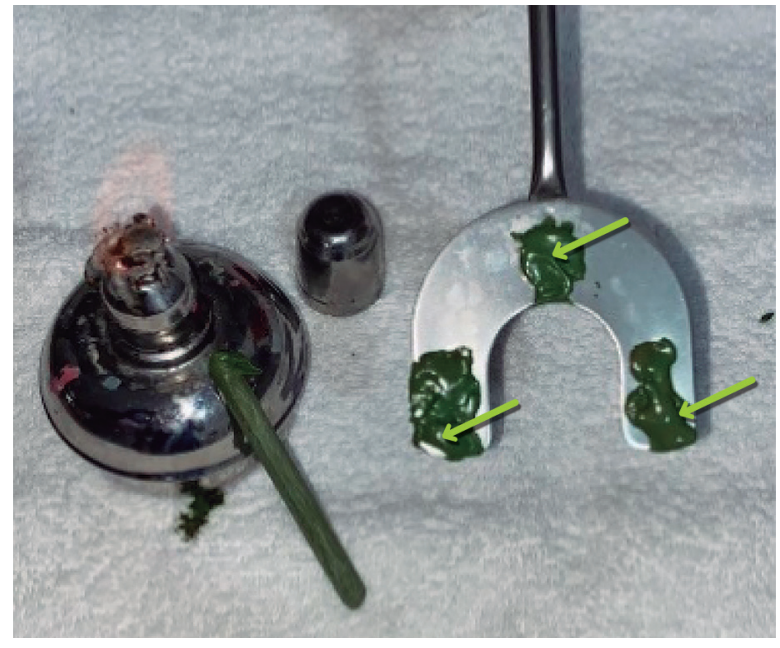

FIGURA 2. Aplicación de godiva.

Fuente: elaboración propia.

\section{PASO N. ${ }^{\circ} 3$}

Llevamos la horquilla a la boca del paciente verificando que quede la línea media de sus dientes en la parte central, hacemos presión sobre sus superficies incisales de los anteriores y oclusales de los posteriores, aseguramos estabilidad de la horquilla (ver figura 3).
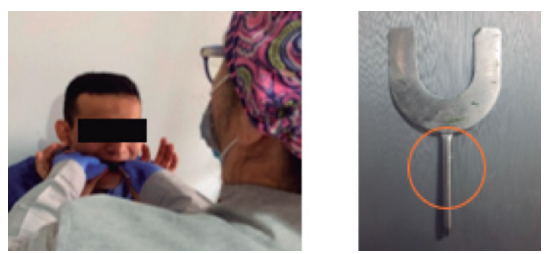

FIGURA 3. Horquilla centrada.

Fuente: elaboración propia. 


\section{PASO N. ${ }^{0} 4$}

Le solicitamos al paciente la colaboración de sostener la horquilla con sus dedos pulgares, como se ve en la figura 4.

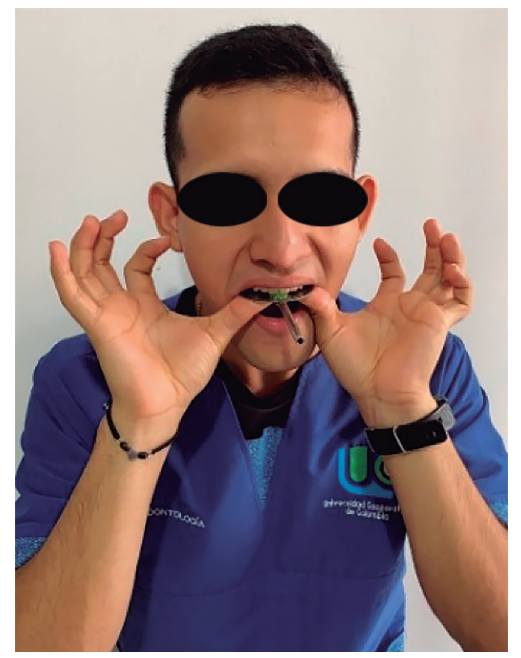

FIGURA 4. Sostener horquilla.

Fuente: elaboración propia

\section{PASO N. ${ }^{0} 5$}

- Procedemos a instalar el arco facial.

- Aflojamos todos los tornillos.

- Instalamos las olivas en los conductos auditivos.

- Le pedimos al paciente que con sus dedos índice y central ajuste a sus oídos las ojivas. Todo lo anterior se ve en la figura 5.
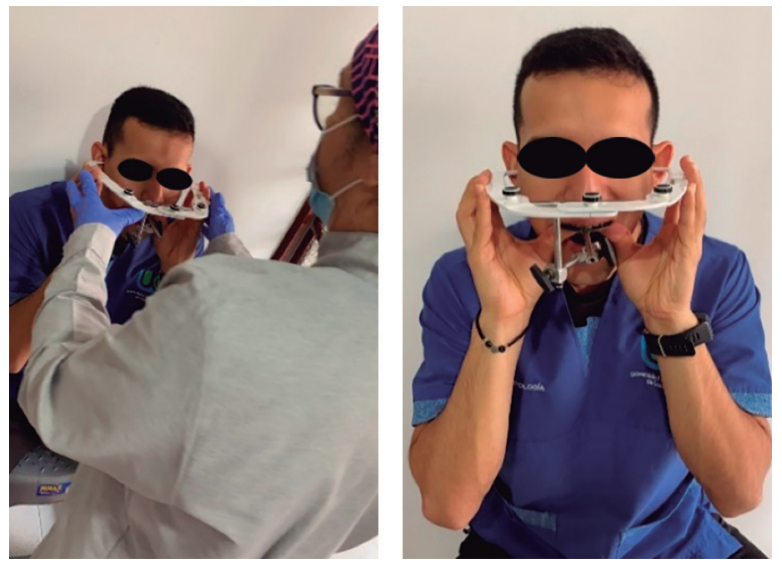

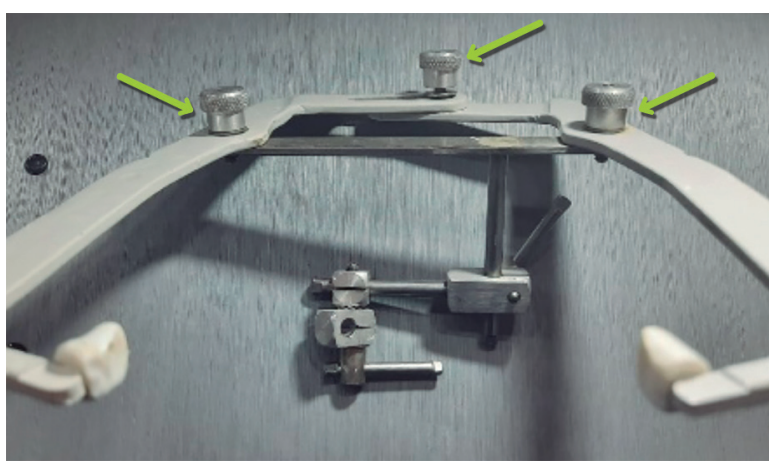

FIGURA 5. Instalación arco facial.

Fuente: elaboración propia

\section{PASO N. ${ }^{\circ} 6$}

Ajustamos los tornillos de ajuste de olivas.
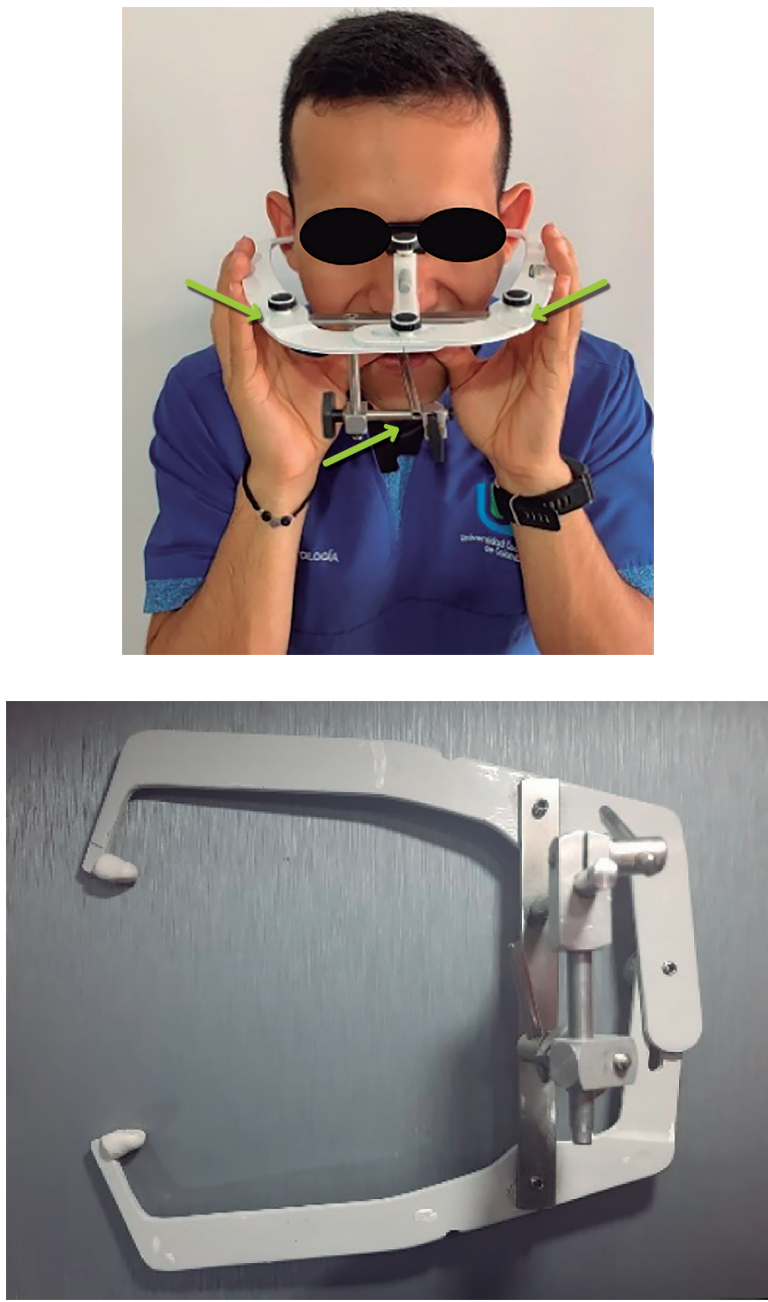

FIGURA 6. Ajuste olivas.

Fuente: elaboración propia. 


\section{PASO N. ${ }^{\circ}$ ?}

Ajustamos el Nasion que es el responsable de suministrar unos de los puntos del tripoidismo.
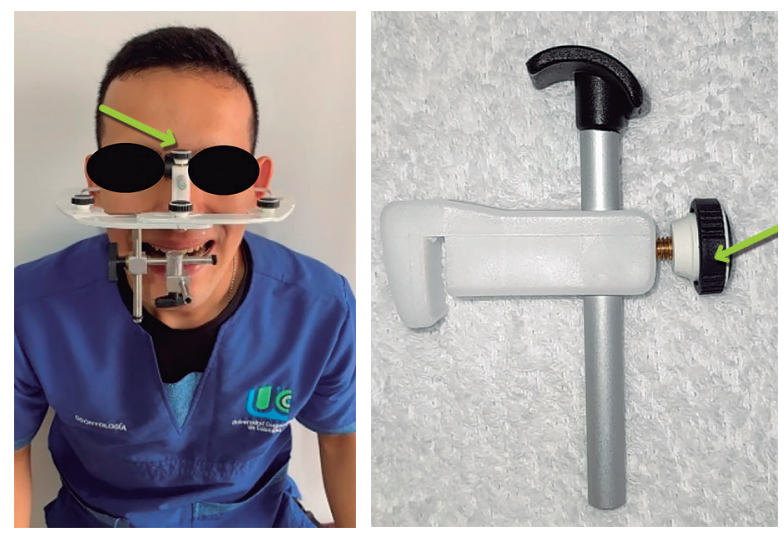

FIGURA ?. Ajuste Nasion.

Fuente: elaboración propia.

\section{PASO N. ${ }^{\circ} 8$}

- Continuamos con el ajuste de los demás tornillos.

- El vástago será el que nos dará la altura del tercio medio y dimensión vertical del paciente.

- Ajustamos el tornillo (ver figura 8).
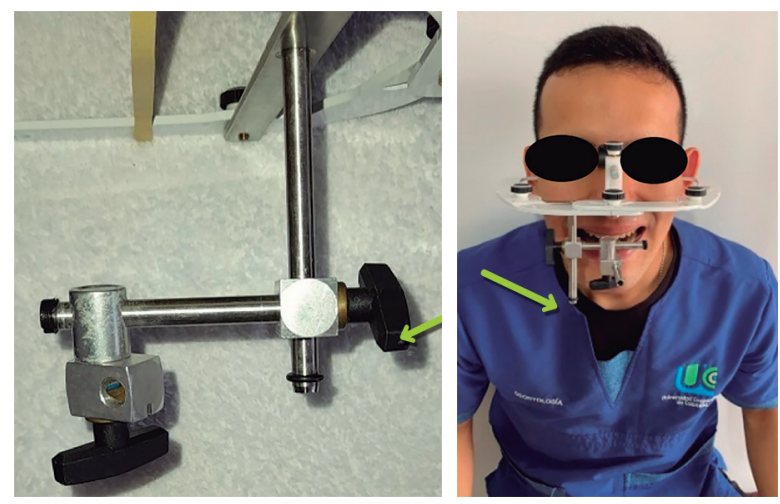

FIGURA 8. Ajuste tornillo del vástago.

Fuente: elaboración propia.

\section{PASO N. ${ }^{\circ} 9$}

- Ajuste de horquilla en la parte inferior.

- El paciente debe soltar lo que venía sosteniendo (figura 9).
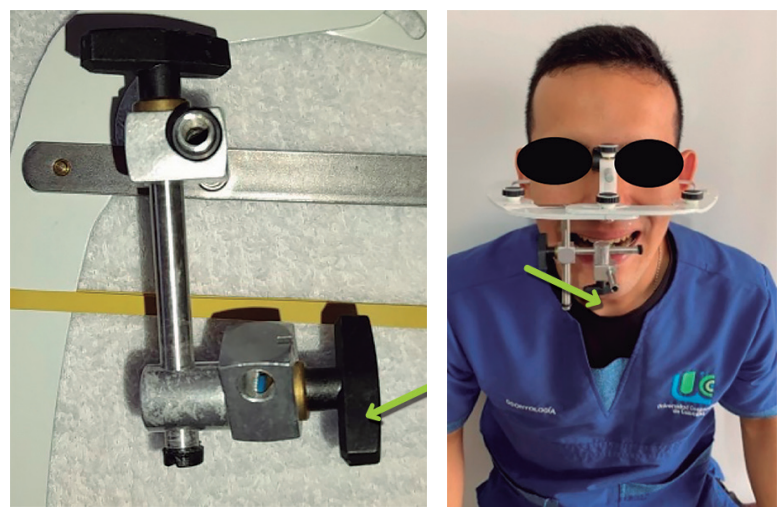

FIGURA 9. Horquilla estable.

Fuente: elaboración propia.

\section{PASO N. ${ }^{\circ} 10$}

El arco debe quedar estable en la cavidad oral del paciente.
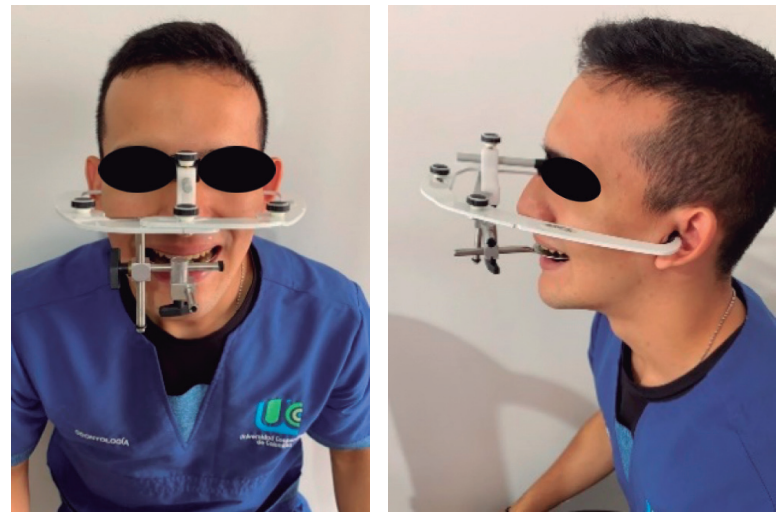

FIGURA 10. Resultado final.

Fuente: elaboración propia. 


\section{Conclusiones}

Una vez examinada esta guía, el estudiante entenderá la demostración que el profesor efectuará y cada uno podrá demostrar el aprendizaje construido desde el inicio del ejercicio, basado en los conocimientos básicos del articulador y su dominio. Al tomar la relación bicondilo maxilar, entendería la posición del maxilar superior con respecto a la base del cráneo para ser trasladado posteriormente al articulador semiajustable.

De esta manera, se alcanza así la competencia requerida: "Analiza las relaciones oclusales en normalidad de la dentición permanente", logro que se alcanza con el análisis del montaje en el articulador, previa toma de la relación bicondilo maxilar.

\section{Referencias}

[1] Insignares, S. Oclusión. 2019. Disponible en http://www.digitac3d.com/articuladores-en-odontolog\%C3\%ADa.html

[2] Leal-Fonseca AP, Hernández-Molinar Y. Evolución de la odontología. Oral. 2016; 17(55):1418-1426. Disponible en https://www.medigraphic.com/cgi-bin/new/resumen.cgi?IDARTICULO=78110

[3] Maldonado Moreno JA, Lombard Romero L, Gutiérrez Camacho C, Canseco Jiménez JF, Cuairán Ruidíaz V. Evaluación de dos técnicas para el registro de relación céntrica mandibular: arco gótico versus céntrica de poder. Rev. Odont. Mex [revista en la Internet]. 2015 Mar [citado 2021 Jun 22]; 19(1): 15-26. Disponible en: http://www.scielo.org.mx/scielo.php?script=sci_arttext\&pid=S1870-199X2015000100003\&lng=es 


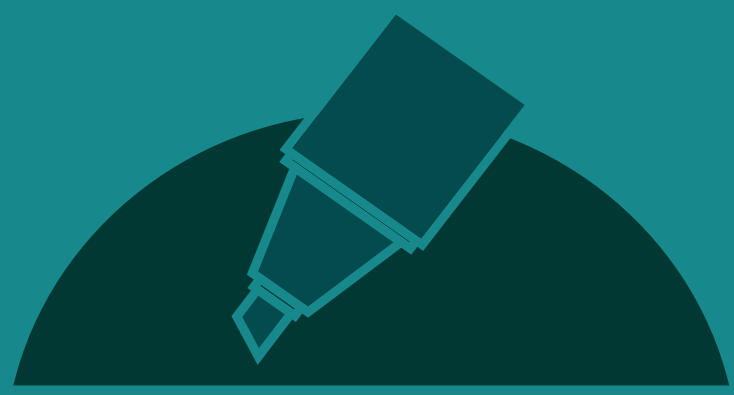

Guías prácticas 